\title{
Fluticasone propionate reduces bacterial airway epithelial invasion
}

\author{
M. Barbier*, A. Agustí ${ }^{\prime, \uparrow,+}$ and S. Albertí*
}

ABSTRACT: Fluticasone propionate reduces the frequency and severity of the episodes of exacerbation of chronic obstructive pulmonary disease (COPD). Streptococcus pneumoniae and Haemophilus influenzae are frequently isolated in these episodes. Both express phosphorylcholine, an epitope that mediates their interaction with airway epithelial cells via the platelet-activating factor receptor (PAFR).

The present work studies the effects of fluticasone propionate on the expression of PAFR on human airway epithelial cells, the invasion of these cells by $S$. pneumoniae and $H$. influenzae, and the course of pneumococcal infection in vivo. The following were used in the experiments: $S$. pneumoniae and $\boldsymbol{H}$. influenzae isolated from patients with COPD, cell cultures of type II pneumocytes and bronchoepithelial cells, and a mouse model of lung infection.

Fluticasone propionate was found to reduce PAFR expression on the surface of the two cells types studied. All S. pneumoniae and $\boldsymbol{H}$. influenzae isolates expressed phosphorylcholine. Treatment of both cells lines with fluticasone propionate reduced invasion of both microorganisms and reduced the bacterial load of mice infected with $S$. pneumoniae.

Fluticasone propionate reduces the invasion of airway epithelial cells by Streptococcus pneumoniae and Haemophilus influenzae through its effect on platelet-activating factor receptor. These results may help explain the beneficial effects of fluticasone propionate on chronic obstructive pulmonary disease exacerbations.

KEYWORDS: Airway epithelial cells, bacterial infections, chronic obstructive pulmonary disease, fluticasone

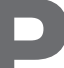
atients with chronic obstructive pulmonary disease (COPD) often suffer episodes of exacerbations of COPD during the course of the disease [1]. These episodes impact negatively on health status, worsen prognosis and are extremely costly. Treatment with inhaled steroids (alone or in combination with long-acting $\beta_{2}$-agonists) reduces the number of exacerbations of COPD [2], particularly in the subgroup of patients with moderate-to-severe disease $[2,3]$ in whom pulmonary inflammation is enhanced [4].

Streptococcus pneumoniae and Haemophilus influenzae are frequently isolated in airway secretions of patients with COPD, particularly during exacerbations [5]. Phosphorylcholine is a surface epitope of S. pneumoniae and H. influenzae $[6,7]$ that binds specifically to the high-affinity plateletactivating factor receptor (PAFR) expressed on the surface of, among other cells types, airway epithelial cells [8]. The interaction between phosphorylcholine and PAFR is a key pathogenic element of $S$. pneumoniae and $H$. influenzae infections $[9,10]$. This is demonstrated by the fact that PAFR-deficient mice are more resistant to pneumococcal pneumonia than wild-type mice [11] and that intratracheal administration of $S$. pneumoniae and a PAFR antagonist to rabbits reduces bacterial loads in lung lavages [9]. Likewise, phosphorylcholine favours the persistence of $H$. influenzae in the airway epithelia and promotes the formation of biofilms $[12,13]$.

Given that the expression of PAFR is elevated in chronically inflamed airways $[9,10]$, such as those found in COPD patients, the present authors hypothesised that steroids may reduce exacerbations by downregulating PAFR expression. To test this hypothesis, the in vitro effect of fluticasone propionate on the following was investigated: 1) the expression of PAFR on airway epithelial cells; 2) the invasion of airway epithelial cells by $S$. pneumoniae or $H$. influenzae; and 3) the course of pneumococcal infection in vivo.

\section{MATERIALS AND METHODS}

\section{Bacterial isolates and reagents}

S. pneumoniae and $H$. influenzae strains were isolated from spontaneous sputum samples from patients with stable and exacerbated COPD.

\section{AFFILIATIONS}

*University Institute of Health Sciences Research (IUNICS), University of the Balearic Islands, \#Pneumology Dept, Son Dureta Hospital,

${ }^{+}$CIBER Enfermedades Respiratorias (CIBERES), Palma de Mallorca, and "The Caubet-Cimera Foundation, Buñola, Spain.

\section{CORRESPONDENCE}

S. Albertí

Institut Universitari dlnvestigacions en Ciències de la Salut (IUNICS)

Universitat de les IIles Balears (UIB) Palma de Mallorca

Spain

Fax: 34971173184

E-mail: sebastian.alberti@uib.es

\section{Received:}

February 112008

Accepted after revision:

July 142008

\section{SUPPORT STATEMENT}

This study has been supported by the Spanish Network for the Research in Infectious Diseases (REIPI RD06/ 0008) and by an unrestricted educational grant from GlaxoSmithKline.

\section{STATEMENT OF INTEREST}

Statement of interest for A. Agustí and S. Albertí, and for the study itself can be found at

www.erj.ersjournals.com/misc/ statements.shtml 
Bacterial isolates were identified and maintained following standard techniques. PAFR antagonist (1-O-Hexadecyl-2acetyl-sn-glycerol-3-phospho-(N,N,N-trimethyl)-hexanolamine) was purchased from Calbiochem (San Diego, CA, USA). The fluticasone propionate was kindly provided by GlaxoSmithKline (London, UK).

\section{Cell culture and treatments}

The human bronchoepithelial immortalised cells 16HBE14oand the human lung carcinoma cells A549 (ATCC CCL185) derived from type-II pneumocytes were used in the present study. The cells were propagated in Earl's minimal essential medium plus $1 \%$ L-glutamine culture medium and in RPMI 1640 plus 1\% HEPES (hydroxyethyl piperazine ethane sulphonic acid), respectively, supplemented with $10 \%$ foetal calf serum and $1 \%$ penicillin-streptomycin (Sigma Aldrich, St Louis, MO, USA). Cells were incubated in 24-well tissue culture plates at $37^{\circ} \mathrm{C}$ and $5 \% \quad \mathrm{CO}_{2}$ until confluence was reached $\left(\sim 5 \times 10^{5}\right.$ cells per well) and then used in the experiments. Monolayers of each cell line were treated at $37^{\circ} \mathrm{C}$ with fluticasone propionate $(0.1 \mu \mathrm{M}, 4 \mathrm{~h})$ or PAFR antagonist $(0.05 \mu \mathrm{M}, 30 \mathrm{~min})$, which were both dissolved in the cell culture medium. The invasion assays were performed as previously described [14]. Briefly, after the incubation of the epithelial cells with the bacterial suspension, the wells were washed with PBS and incubated with fresh medium containing gentamicin $\left(100 \mu \mathrm{g} \cdot \mathrm{mL}^{-1}\right)$ to kill extracellular bacteria. After $1 \mathrm{~h}$, an aliquot of the medium was plated to confirm killing of extracellular bacteria, and the gentamicin-containing medium was rewashed. The epithelial cells were lysed and intracellular bacteria were quantified by plating appropriate dilutions on blood agar plates.

\section{Flow cytometry}

Monolayers of treated cells were detached with $0.25 \%$ trypsinEDTA (Sigma Aldrich), washed with PBS and fixed for $18 \mathrm{~h}$ at $4^{\circ} \mathrm{C}$ with $1 \%$ paraformaldehyde in PBS. Cells were then washed, resuspended at a concentration of $5 \times 10^{4}$ cells $\cdot \mathrm{mL}^{-1}$ in PBS-1\% bovine serum albumin (BSA), and incubated for $3 \mathrm{~h}$ at $25^{\circ} \mathrm{C}$ with rabbit anti-PAFR diluted at 1:500 (Alexis Biochemicals, San Diego, CA, USA). After washing, cells were incubated for $1 \mathrm{~h}$ at $25^{\circ} \mathrm{C}$ with goat anti-rabbit immunoglobulin (Ig)G conjugated fluorescein isothiocyanate diluted at 1:500. Finally, cells were resuspended in isoton buffer for analysis. Analysis was carried out in an Epics XL flow cytometer using the Expo32 software (both Beckman Coulter, Madrid, Spain). Nonspecific binding was corrected with the corresponding isotype-matched antibodies.

\section{Immunoblot assay}

Phosphorylcholine expression by the bacterial isolates was assessed by immunoblotting, as described previously [7]. Bacterial suspensions were spotted on to nitrocellulose membranes. After blocking in PBS-1\% BSA, membranes were sequentially incubated with specific monoclonal antibody $(\mathrm{mAb})$ against phosphorylcholine (TEPC-15; Sigma Aldrich) and a goat anti-mouse IgA/alkaline phosphatase conjugate (Caltag Laboratories, Burlingame, CA, USA). Washing with PBS was included after incubations. Alkaline phosphatase was visualised on the blots using a BCIP/NBT fast kit (Sigma Aldrich). Finally, the filters were analysed by densitometry using a BioImage densitometer and Whole band 3.1 software (both Millipore, Barcelona, Spain). Klebsiella pneumoniae, a microorganism that does not present phosphorylcholine on its surface [15], was used as a negative control.

\section{Murine model of lung infection}

Male (20-25 g) ICR-CD1 mice (Harlan Ibérica, Barcelona, Spain) were anesthetised and inoculated intratracheally with $10^{6}$ colony-forming units of S. pneumoniae using a blunt-end feeding needle. Pneumococci were inoculated with saline or saline solutions of PAFR antagonist $(0.25 \mu \mathrm{g})$ or fluticasone propionate ( $10 \mu \mathrm{g} ; \mathrm{n}=16$ per group). At 24 and $48 \mathrm{~h}$, eight animals of each group were sacrificed. The lungs were aseptically removed and homogenised for quantitative bacterial cultures by plating appropriate dilutions on blood agar plates [14]. All animal experiments were carried out according to institutional and national guidelines and approved by the Experimental Animal Committee of the Universitat Illes Balears (Palma de Mallorca, Spain).

\section{Statistical analysis}

All values are presented as mean \pm SEM. A two-tailed unpaired t-test was used to compare the effect of each treatment with the control. A p-value of $\leqslant 0.05$ was considered significant.

\section{RESULTS}

Fluticasone propionate reduces PAFR expression on airway epithelial cells.

Pneumocytes type-II derived cells A549 and bronchoepithelial cells 16HBE14o- constitutively expressed PAFR (fig. 1). Treatment with the PAFR antagonist reduced the expression of the receptor in both cell types compared with untreated cells $(\mathrm{p}<0.05$ for each cell type versus control). Interestingly, fluticasone propionate also reduced the expression of PAFR in A549 and 16HBE14o- ( $p<0.05$ for each cell type versus control).

\section{Effect of fluticasone propionate on the invasion of airway epithelial cells by S. pneumoniae and $\mathrm{H}$. influenzae}

The presence of phosphorylcholine on the surface of the various $S$. pneumoniae and $H$. influenzae isolates from COPD patients was investigated first, as the expression of phosphorylcholine undergoes phase variation in both microorganisms $[12,16]$. This was examined using immunoblot analysis with the $\mathrm{mAb}$ TEPC-15, which is highly specific for the phosphorylcholine epitope [17]. All S. pneumoniae and H. influenzae isolates reacted with TEPC- 15 but a wide variability in the expression of phosphorylcholine was observed among different isolates of either S. pneumoniae or H. influenzae (fig. 2).

The effect of fluticasone propionate on the invasion of the epithelial cells by both microorganisms was studied by selecting one strain of each species in which the phosphorylcholine expression was intermediate (fig. 2). In preliminary experiments, it was observed that neither PAFR antagonist nor fluticasone propionate affected bacteria or cell viability. The effect of different fluticasone propionate doses $(0.05,0.1$ and $0.5 \mu \mathrm{M})$ on the invasion of either $H$. influenzae and S. pneumoniae to A549 cells was also tested. All concentrations tested reduced the invasion of both microorganisms in a dosedependent manner (data not shown). The intermediate concentration $(0.1 \mu \mathrm{M})$ was selected for further experiments because it was the lowest concentration that produced the best 

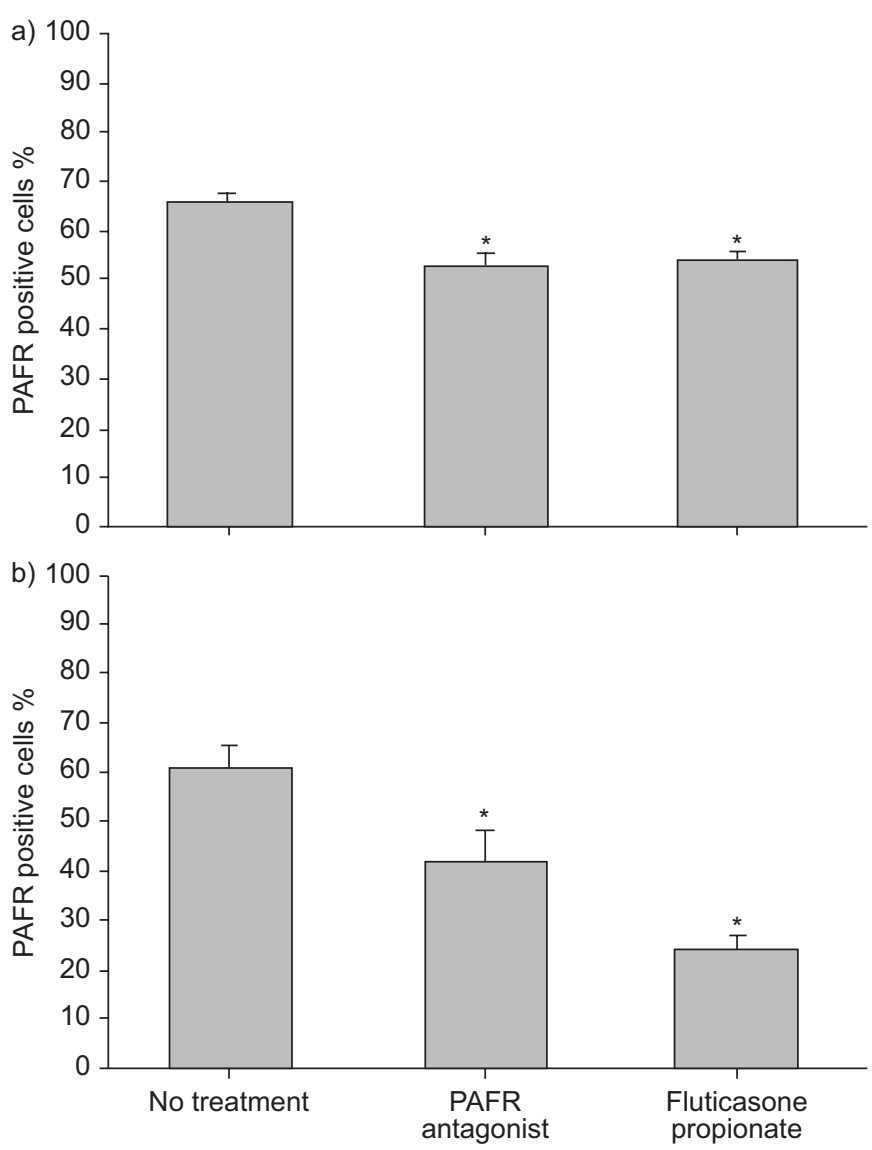

FIGURE 1. The effect of fluticasone propionate on the expression of plateletactivating factor receptor (PAFR) on the airway epithelial cells surface. a) A549 and b) 16HBE140- cells were treated with PAFR antagonist ( $0.05 \mu \mathrm{M}$ for $30 \mathrm{~min})$ or fluticasone propionate $(0.1 \mu \mathrm{M}$ for $4 \mathrm{~h})$. The cell surface-associated PAFR was determined by flow cytometry analysis using a monoclonal antibody, anti-PAFR. The results are from five independent experiments. ${ }^{*}: p<0.05$ versus untreated cells.

results for treatment comparison. As shown in figure 3, treatment of monolayers of both types of airway epithelial cells with $0.1 \mu \mathrm{M}$ of fluticasone propionate significantly reduced the invasion of $H$. influenzae and S. pneumoniae with respect to the untreated control $(\mathrm{p}<0.05$ for each pathogen and cell type versus control). Invasion was reduced between 60 to $28 \%$ depending on the microorganism and the epithelial cell type. PAFR antagonist was used as a positive control and, as expected, reduced the capacity to invade the epithelial cells of both microorganisms.

\section{Effect of fluticasone propionate on the lung colonisation of S. pneumoniae in vivo}

Fluticasone propionate administered at $10 \mu \mathrm{g}$ per mouse reduced pneumococcal lung colonisation by almost $50 \%$ at $24 \mathrm{~h}$ and $48 \mathrm{~h}$ after infection ( $\mathrm{p}<0.05$ for each group versus control; fig. 4). As previously described [9], the inoculation of PAFR antagonist also significantly reduced the pneumococcal load of the infected animals.

\section{DISCUSSION}

The main findings of the present study were that fluticasone propionate reduces: 1) the expression of PAFR in human lung

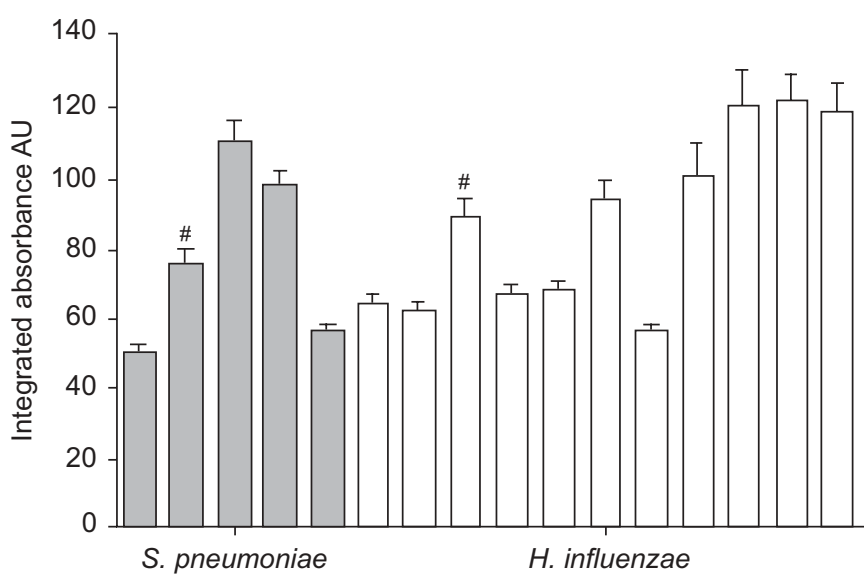

FIGURE 2. Analysis of the phosphorylcholine expression on Streptococcus pneumoniae or Haemophilus influenzae isolates from chronic obstructive pulmonary disease (COPD) patients. Bacterial cells from COPD patients' isolates were spotted on membranes and phosphorylcholine was detected with the specific monoclonal antibody TEPC-15 (see Materials and Methods section). Quantification was carried out by densitometric analysis of the spots using a Biolmage densitometer and Whole band 3.1 software (both Millipore, Barcelona, Spain). Data are presented as mean \pm SD of three independent experiments. Control values, such as phosphorylcholine expression by Klebsiella pneumoniae, were removed from experimental

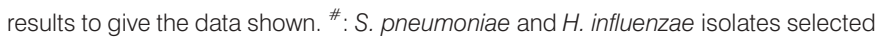
for further experiments.

epithelial cells; 2) the invasion of these cells by $H$. influenzae and S. pneumoniae; and 3) pneumococcal lung colonisation in vivo. These experimental findings can be relevant for the observed effects of fluticasone propionate in COPD.

It is generally accepted that bacterial infection/colonisation is a key factor in the pathogenesis of COPD exacerbations. Interestingly, S. pneumoniae and H. influenzae, which are frequently isolated in patients with COPD exacerbations [15, 18], share a common epitope, phosphorylcholine. This bacterial surface epitope mediates the interaction of these pathogens with PAFR, which is present on airway epithelial cells. The phosphorylcholine-PAFR interaction is critical for the pathogenesis of infections by both pathogens $[9,10]$. The present results show that fluticasone propionate reduces the expression of PAFR in two predominant cell types of the airway epithelia. As a consequence, the capacity of both pathogens to invade the epithelial cells is impaired. DowLING et al. [19] reported that fluticasone propionate also has the ability to reduce the total number of Pseudomonas aeruginosa that adhere to the respiratory mucosa. Dowling et al. [19] suggested that this reduction was due to a decrease in the amount of mucosal damage caused by the microorganism, to which the bacteria preferentially adhered. According to the present results, it can be hypothesised that downregulation of PAFR expression might also have contributed to the effects of fluticasone propionate on the adherence of $P$. aeruginosa to respiratory mucosa, as this respiratory pathogen also expresses phosphorylcholine [20]. The current results also support previous studies that described the key role of the bacterial epitope, phosphorylcholine, in the interaction of the respiratory pathogens with the host cells $[9,10]$. However, phosphorylcholine expression undergoes phase variation in $S$. pneumoniae and $H$. influenzae $[12,16]$. The fact that all isolates 

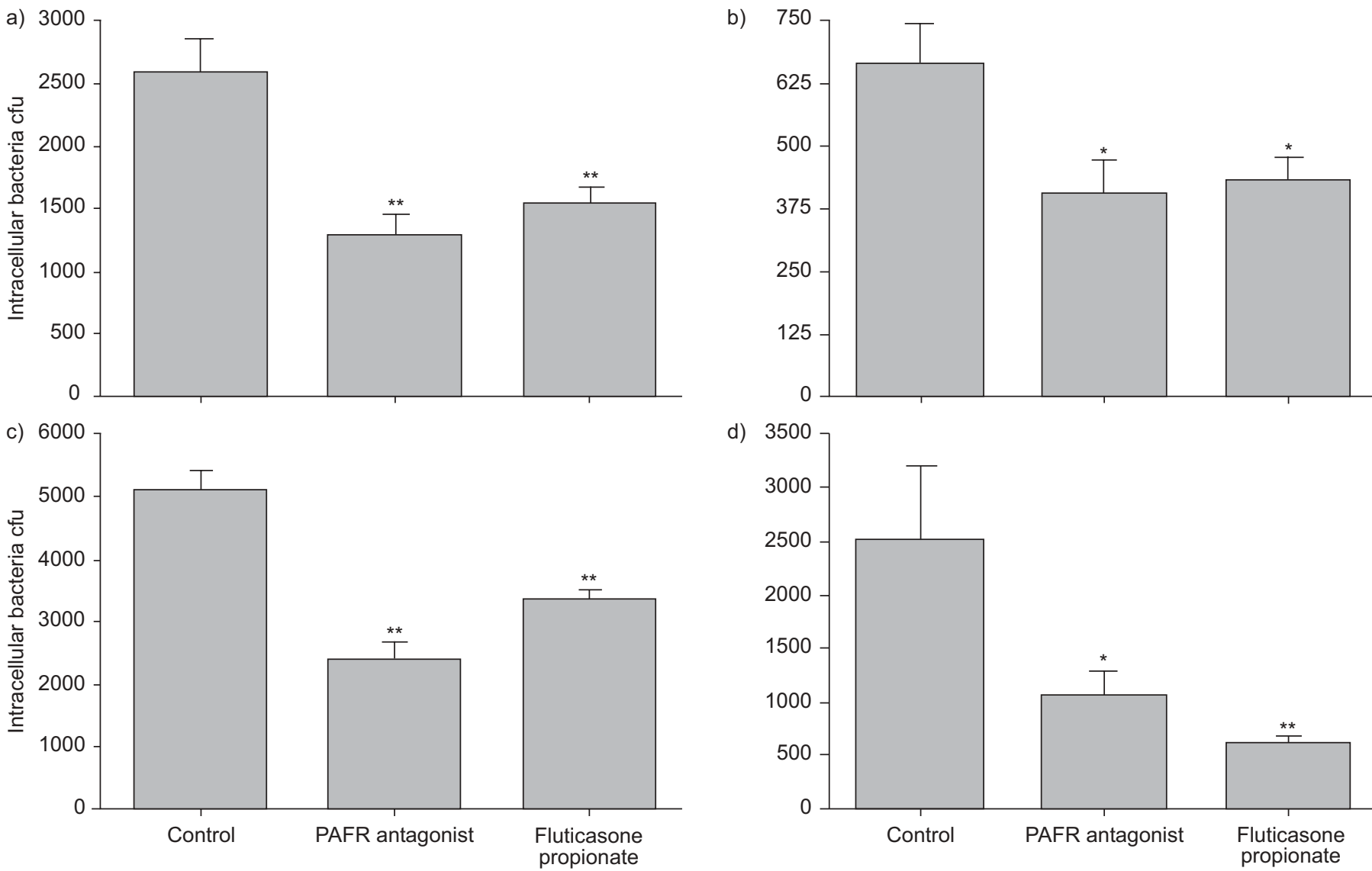

FIGURE 3. The effect of fluticasone propionate on the invasion of the airway epithelial cells by Streptococcus pneumoniae and Haemophilus influenzae. a, c) Monolayers of A549 cells and b, d) 16HBE14o- cells were treated with platelet-activating factor receptor (PAFR) antagonist or fluticasone propionate and incubated with $10^{7}$ colonyforming units (cfu) of $\mathrm{S}$. pneumoniae ( $\mathrm{a}$ and $\mathrm{b}$ ) or $\mathrm{H}$. influenzae ( $\mathrm{c}$ and d). Intracellular bacteria was determined as described in the Materials and Methods section. Data are presented as mean \pm SEM of five independent experiments. *: $p<0.05$ versus controls; **: $p<0.01$ versus controls.

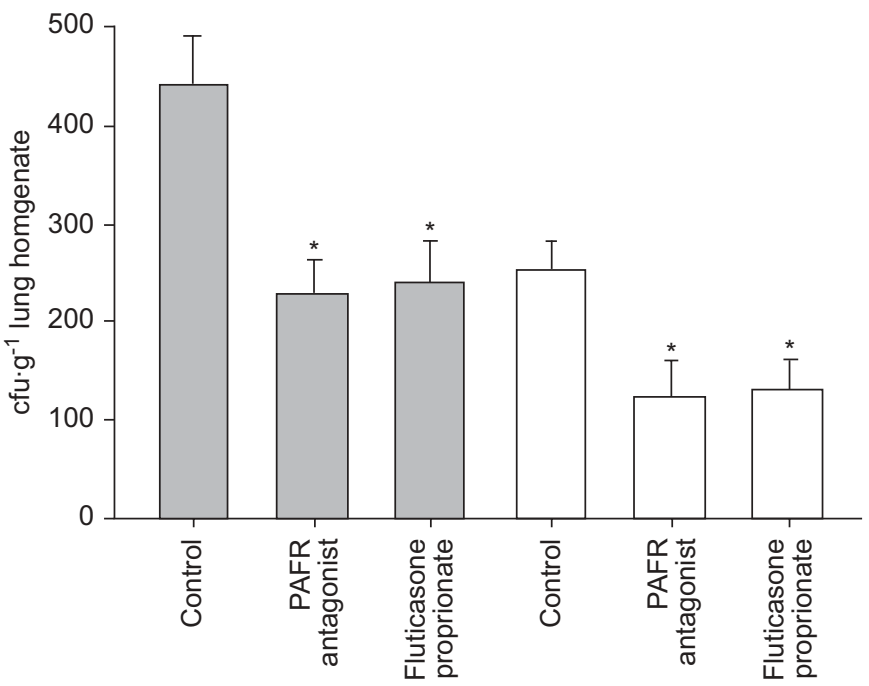

FIGURE 4. The effect of fluticasone propionate on the Streptococcus pneumoniae lung infection in vivo. Mice were intratracheally inoculated with $S$. pneumoniae resuspended with saline (control) or with saline solutions containing platelet-activating factor receptor (PAFR) antagonist or fluticasone propionate. The number of bacterial cells $\cdot g^{-1}$ of lung homogenate was determined at $24(\square)$ and $48 \mathrm{~h}(\square)$. cfu: colony-forming unit. *: $p<0.05$ versus controls. tested in the present study expressed phosphorylcholine on their outer surfaces suggests that phosphorylcholine may represent an important adaptation to the abnormal lung environment encountered by $S$. pnemoniae and $H$. influenzae in the airways of COPD patients.

The present study found that airway epithelial cells express PAFR constitutively. CUNDELL et al. [9] showed that activation of airway epithelial cells with interleukin-1 upregulates the surface expression of PAFR which, in turn, facilitates the invasion by $S$. pneumoniae via the interaction between PAFR and the phosphorylcholine component of the bacterial surface. To the current authors' knowledge, there are no data in the literature reporting the level of expression of PAFR on airway epithelial cells of patients with COPD. However, it is conceivable that the chronic inflammatory response that characterises COPD [4] can upregulate the expression. In fact, this is the case for other receptors involved in the interaction with bacterial pathogens, such as the protein G-coupled receptor intercellular adhesion molecule-I [21] or Toll-like receptor-2 [22].

The present study did not investigate the molecular mechanism that mediates the reduction of PAFR due to fluticasone propionate. However, several nonexclusive mechanisms can 
be predicted. First, fluticasone propionate can reduce PAFR expression indirectly [9] through the reduction of lung inflammation [1]. Secondly, fluticasone propionate can increase the synthesis of lipocortin, which in turn inhibits the production of lipid phospholipase A and, hence, the production of lipid mediators such as leukotrienes, prostaglandins and platelet-activating factor [23]. Finally, fluticasone propionate can prevent airway mucosal damage [19]. These mechanisms alone or in combination can explain the current in vivo observations. However, further studies are required to establish the molecular and cellular basis of the effect of fluticasone propionate on the PAFR expression in vitro and in vivo.

The present study demonstrates that fluticasone propionate reduces PAFR expression on airway epithelial cells, and that it prevents the invasion of S. pneumoniae and H. influenzae in vitro and in vivo. These novel molecular effects can explain the reduction in the frequency of exacerbations observed in patients treated with fluticasone propionate. They seem to differ to the recently reported increase in pneumonia in the same patients [24]. Several nonexclusive factors might explain this apparent discrepancy. First, in the previous study the diagnosis of pneumonia in patients treated with fluticasone propionate was often not confirmed by chest radiography [24]. Thus, the accuracy of a clinical diagnosis of "pneumonia" is questionable. Secondly, no attempt was made to obtain a specific microbiological diagnosis in these patients in the previous study [24], and several bacteria and viruses may have accounted for such pneumonia. In contrast, in the present study, the effect of fluticasone propionate was tested using two well-characterised bacterial pathogens. Finally, the current results indicate that fluticasone propionate reduces bacterial load during the first steps of pulmonary infection, but the results do not exclude the possibility that fluticasone propionate may also inhibit the recruitment of phagocytes to the lung, thus increasing the susceptibility to pneumonia. In this context, it is worth mentioning that the reduced bacterial invasion of the epithelial cells induced by fluticasone propionate may also reduce the expression of certain inflammatory cytokines, which might be critical for the recruitment of phagocytes to the infection site.

In conclusion, the current results demonstrate that fluticasone propionate reduces the invasion of the airway epithelial cells by Streptococcus pneumoniae and Haemophilus influenzae. However, further studies are required to understand the biological significance of this phenomenon, particularly in the context of the airway infection/colonisation that often occurs in patients with chronic obstructive pulmonary disease.

\section{ACKNOWLEDGEMENTS}

The authors would like to thank T. de Francisco (Scientific/ technical services, Universitat de les Illes Balears, Palma de Mallorca, Spain) for assistance with the animal experiments.

\section{REFERENCES}

1 Global Initiative for Chronic Obstructive Lung Diseases. Global Strategy for the Diagnosis, Management, and Prevention of Chronic Obstructive Pulmonary Disease, 2006. www.goldcopd.com Date last accessed: June 2008. Date last updated: January 2008.
2 Burge PS, Calverley PMA, Jones PW, Spencer S, Anderson JA, Maslen TK. Randomised, double blind, placebo controlled study of fluticasone propionate in patients with moderate to severe chronic obstructive pulmonary disease: the ISOLDE trial. BMJ 2000; 320: 1297-1303.

3 Sin DD, Wu L, Anderson JA, et al. Inhaled corticosteroids and mortality in chronic obstructive pulmonary disease. Thorax 2005; 60: 992-997.

4 Hogg JC, Chu F, Utokaparch S, et al. The nature of smallairway obstruction in chronic obstructive pulmonary disease. N Engl J Med 2004; 350: 2645-2653.

5 Sethi S, Murphy TF. Bacterial infection in chronic obstructive pulmonary disease in 2000: a state-of-the-art review. Clin Microbiol Rev 2001; 14: 336-363.

6 Fischer W. Phosphocholine of pneumococcal teichoic acids: role in bacterial physiology and pneumococcal infection. Res Microbiol 2000; 151: 421-427.

7 Weiser JN, Shchepetov M, Chong STH. Decoration of lipopolysaccharide with phosphorylcholine: a phase-variable characteristic of Haemophilus influenzae. Infect Immun 1997; 65: 943-950.

8 Ishii S, Shimizu T. Platelet-activating factor (PAF) receptor and genetically engineered PAF receptor mutant mice. Prog Lipid Res 2000; 39: 41-82.

9 Cundell DR, Gerard NP, Gerard C, Idanpaanheikkila I, Tuomanen EI. Streptococcus pneumoniae anchor to activated human-cells by the receptor for platelet-activating factor. Nature 1995; 377: 435-438.

10 Swords WE, Buscher BA, Li KVS, et al. Non-typeable Haemophilus influenzae adhere to and invade human bronchial epithelial cells via an interaction of lipooligosaccharide with the PAF receptor. Mol Microbiol 2000; 37: 13-27.

11 Rijneveld AW, Weijer S, Florquin S, et al. Improved host defense against pneumococcal pneumonia in plateletactivating factor receptor-deficient mice. J Infect Dis 2004; 189: 711-716.

12 Weiser JN, Pan N, McGowan KL, Musher D, Martin A, Richards J. Phosphorylcholine on the lipopolysaccharide of Haemophilus influenzae contributes to persistence in the respiratory tract and sensitivity to serum killing mediated by C-reactive protein. J Exp Med 1998; 187: 631-640.

13 Hong WZ, Mason K, Jurcisek J, Novotny L, Bakaletz LO, Swords WE. Phosphorylcholine decreases early inflammation and promotes the establishment of stable biofilm communities of nontypeable Haemophilus influenzae strain 86-028NP in a chinchilla model of otitis media. Infect Immun 2007; 75: 958-965.

14 Cortés G, Álvarez D, Saus C, Albertí S. Role of lung epithelial cells in defense against Klebsiella pneumoniae pneumonia. Infect Immun 2002; 70: 1075-1080.

15 Jansen HM, Sachs APE, Vanalphen L. Predisposing conditions to bacterial-infections in chronic obstructive pulmonarydisease. Am J Respir Crit Care Med 1995; 151: 2073-2080.

$16 \mathrm{Kim}$ JO, Weiser JN. Association of intrastrain phase variation in quantity of capsular polysaccharide and teichoic acid with the virulence of Streptococcus pneumoniae. J Infect Dis 1998; 177: 368-377.

17 Leon MA, Young NM. Specificity for phosphorylcholine of six murine myeloma proteins reactive with pneumococcus C-polysaccharide and $\beta$-lipoprotein. Biochemistry 1971; 10: 1424-1429. 
18 Vanalphen L, Jansen HM, Dankert J. Virulence factors in the colonization and persistence of bacteria in the airways. Am J Respir Crit Care Med 1995; 151: 2094-2100.

19 Dowling RB, Johnson M, Cole PJ, Wilson R. Effect of fluticasone propionate and salmeterol on Pseudomonas aeruginosa infection of the respiratory mucosa in vitro. Eur Respir J 1999; 14: 363-369.

20 Barbier M, Oliver A, Rao J, Hanna SL, Goldeberg JB, Albertí S. Novel phosphorylcholine-containing protein of Pseudomonas aeruginosa chronic infection isolates interacts with airway epithelial cells. J Infect Dis 2008; 197: 465-473.
21 Noguera A, Busquets X, Sauleda J, et al. Expression of adhesion molecules and $G$ proteins in circulating neutrophils in chronic obstructive pulmonary disease. Am J Respir Crit Care Med 1998; 158: 1664-1668.

22 Pons J, Sauleda J, Regueiro V, et al. Expression of Toll-like receptor 2 is up-regulated in monocytes from patients with chronic obstructive pulmonary disease. Respir Res 2006; 7: 64.

23 Flower RJ, Rothwell NJ. Lipocortin-1: cellular mechanisms and clinical relevance. Trends Pharmacol Sci 1994; 15: 71-76.

24 Calverley PMA, Anderson JA, Celli B, et al. Salmeterol and fluticasone propionate and survival in chronic obstructive pulmonary disease. $N$ Engl J Med 2007; 356: 775-789. 